\title{
AEROSOL CONCENTRATIONS AND THEIR BEHAVIOUR DURING SUMMER SEASON AT URAWA \\ 1. RELATIONS TO WIND
}

\author{
T. Suda, M. Murai, and N. Kitagawa \\ Faculty of Engineering, Saitama University \\ Urawa-Shi 338
}

\begin{abstract}
The concentrations of small ions, Aitken and Mie particles were measured for 5 months during the summer season and their relations to wind were investigated. The concentrations tend to increase for small ions and to decrease for Mie particles with the increament of the wind speed, while the Aitken particle concentration is rather insensitive for the wind speed. The direction of the wind influences the concentrations of all the 3 types of aerosols, if the strong aerosol sources are located in certain windward directions.
\end{abstract}

\section{Introduction}

From June 1 to October 31, 1980, the continuous measurements of the concentrations of small ions, and Aitken and Mie particles were carried out at Saitama University, Urawa City, in order to investigate the behaviour of aerosols. The effects of the wind on the aerosol concentrations have been discussed by Ikegami et al. (1979, 1980) and Kobayashi et al. (1980). In the present paper, further investigations on relations to wind have been done.

\section{Method of Measurements}

The positive and negative polar conductivities were measured by a Gerdien type conductivity meter. The concentrations of Aitken and Mie particles were measured by a Pollak counter and a photocounting particle meter, respectiveIy. The measurements were carried simultaneously for 10 minutes every 30 minutes in the laboratory room which is located about 15 meters above the ground. For Mie particles, particles the diameter of which is larger than $0.5 \mathrm{~m}$ were analyzed. Since the relative mobility spectrum and hence the effective mobilities are invariable for the small ions under ordinary meteorological conditions, the concentrations of the positive and negative ions were calculated from respective polar conductivities, using the effective mobilities of $1.16 \times 10^{-4} \mathrm{~m}^{2} \mathrm{~V}^{-1} \mathrm{~s}^{-1}$ and $1.30 \times 10^{-4} \mathrm{~m}^{2} \mathrm{~V}^{-1} \mathrm{~s}^{-1}$ for positive and negative ions, the values presented by Misaki (1981). In analying the results, the total conductivities and the sum of the positive and negative small ions were used. The wind speed and direction were measured for 10 minutes hourely at the Saitama Institute of Environmental pollution, which is situated about 500 meters north-east the laboratory.

3. Results

3-1. Relation of the Mie and Aitken particle concentrations to the wind speed.

In the day time, the inverse correlation is always observed between the Mie particle concentration and the wind speed, while at night this correlation fails to hold. Hence, the data measured from 06:00 to 20:00 were analyzed. The frequency distributions of the Mie particle concentration are shown in Fig.1, where the distributions were shown for each wind speed range of $1 \mathrm{~ms}^{-1}$. The concentration increases as the wind speed decreases and high concentration can hardly be seen for the wind speed higher than $4 \mathrm{~ms}^{-1}$. The mode also varies according to the wind speed, e.g. the mode stands for $2 \sim 4$ particles $\mathrm{cm}^{-3}$ for 
the wind speed range of $1 \sim 2 \mathrm{~ms}^{-1}$, and $4 \sim 6$ particles $\mathrm{cm}^{-3}$ for that of $0 \sim 1 \mathrm{~ms}^{-1}$.

Figure 2 shows a plot of the Aitken particle concentration. In contrast with Mie particles, distributions of the Aitken particle concentration scarcely vary over the wind speed range from $0 \sim 1 \mathrm{~ms}^{-1}$ to $6 \sim 7 \mathrm{~ms}^{-1}$, the mode of the concentration always staying between $30 \sim 10$ and $40 \sim 10$ particles $\mathrm{cm}^{-3}$.

Fig.1. Distribution of Mie particle concentration classified by wind speed.

3-2. Relation of the concentrations of 3 types of aerosols to the wind speed and direction

The wind direction was expressed by 16 direction number, 0 standing for the north. The concentrations of 3 types of aerosols were plotted against the direction numbers. For the directions from $3 \sim 10$, the concentrations are little higher for the Aitken particles and little lower for the small ions. No definite feature can be found for the Mie particles.

Fig.2. Distribution of Aitken particle concentration classified by wind speed.

In order to examine the combined effect of the wind speed and direction, the 16 directions were grouped into 4 direction sectors $i . e$. directions $3 \sim 6$, $7 \sim 10,11 \sim 14$ and $15 \sim 2$ into the east, south, west and north direction sectors, respectively. The concentrations averaged for each direction sector were plotted against the wind speed.

Figure 3 shows a plot of small ion concentrations which are observed to increase as the wind speed increases for the west, north and south winds. On the contrary, the concentration slightly decreases for the east wind.
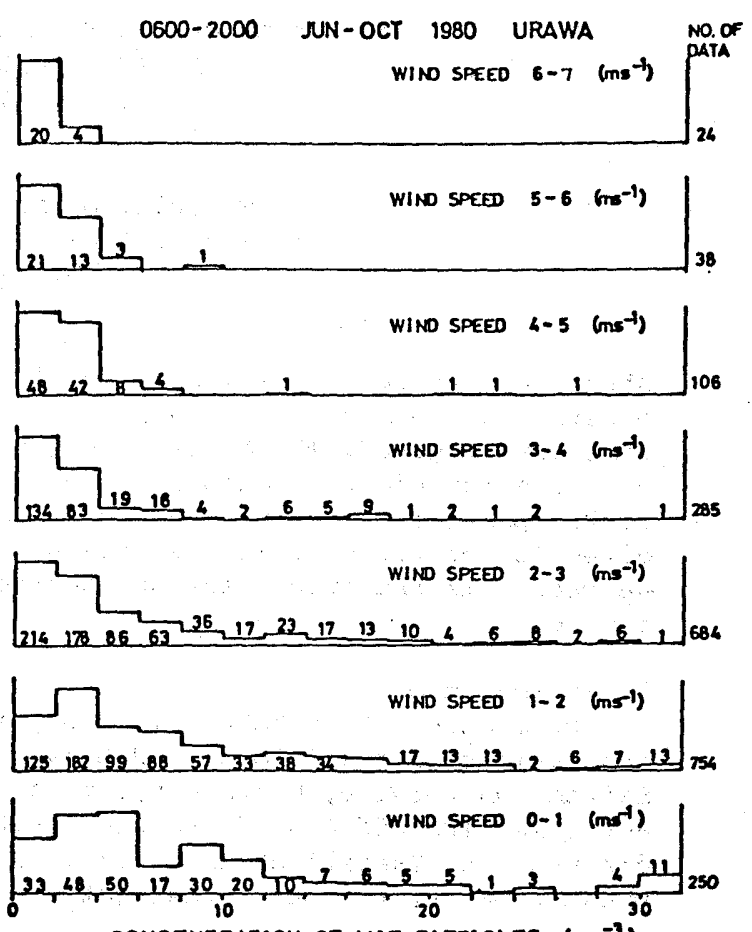

CONCENTRATION OF MIE PARTICLES $\left(\mathrm{cm}^{-3}\right)$
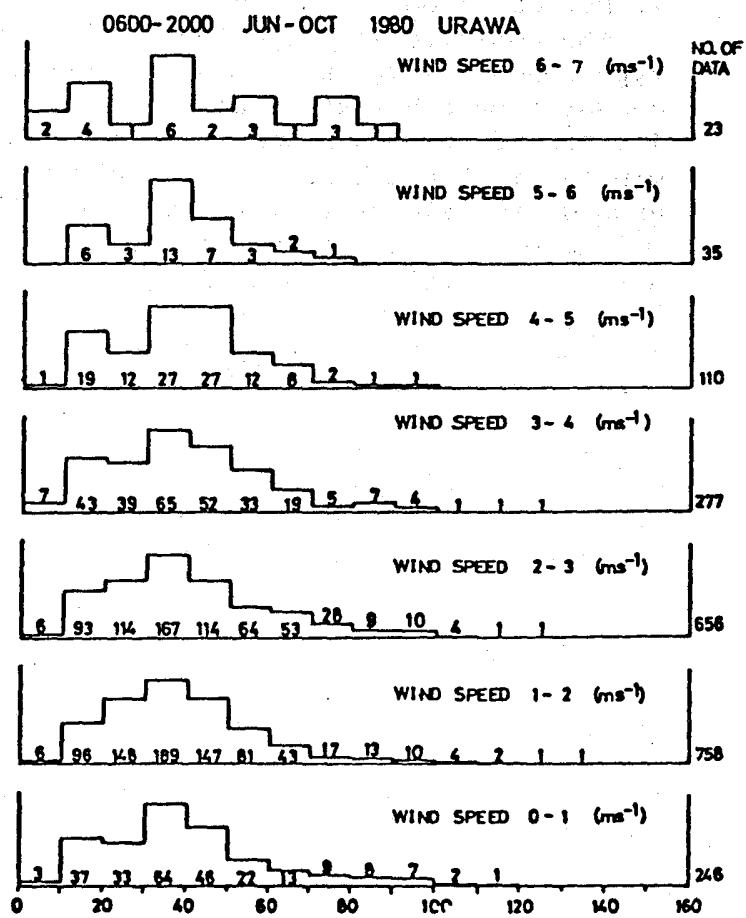

CONCENTRATION OF AITKEN PARTICLES $\left(\times 10^{3} \mathrm{~cm}^{-3}\right)$ 
Figure 4 shows a plot of the Aitken centrations, where the variation against the wind speed is very small. The concentration is observed to increase slightly only for the east wind.

Figure 5 shows a plot of the Mie particle concentrations, where they decrease for all 4 direction sectors, but the decrease for the east wind is less steep than for the other winds.

It is noteworthy that the trends of the concentration variations for east wind are always different from other winds for each 3 types of aerosols, as seen in Figs. $3 \sim 5$.

3-3. Occurrence of the extremely high small ion concentration

The peak of small ion concentration or conductivity was usually observed at night from midnight to sunrize. During the whole observation period, the extremely high peak values were recorded on 17 specific days, where the conductivity was found to exceed $13 \times 10^{-15} \mathrm{Sm}^{-1}$ which stands for 288 ions $\mathrm{cm}^{-3}$. The 17 peak values are plotted against the wind speed in Fig.6. The figure also indicates that these high ion concentrations are associated with strong wind.

In Fig.7. the concentrations of Aitken and Mie particles associated with these high ion concentration peaks are plotted against the small ion concentration. The levels of the concentrations of Aitken and Mie particles during 24:00 05:00 averaged over the whole observation period are also shown by level lines. The figure indicates that all the Mie particle dots situate under the average level, and the Aitken particle dots also situate under the average level except for only 4 dots. It should be noted that in the latter 4 cases, the combined Mie particle dots situate in an extremely low level. On the whole, it is observed that low concentrations of both Aitken and Mie particles combine to result in the high small ion concentration.

\section{Conclusion}

4-1. The results shown in 3-2 are reasonably explained, if the strong aerosol source is assumed to exist in the east of the observation point. Since the center part of Urawa City situates $4 \sim 8 \mathrm{~km}$ to the east of the observation point, this assumption is highly probable, and hence the following

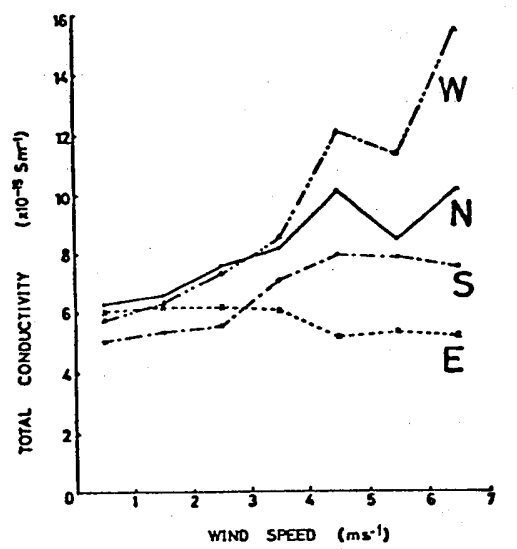

Fig.3. Conductivity versus wind speed for east, south, west and north winds.

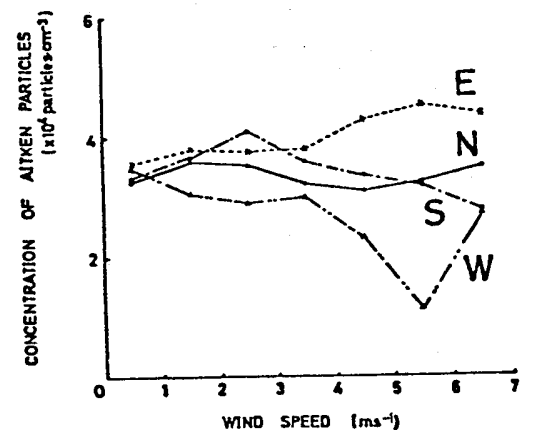

Fig.4. Aitken particle concentrations versus wind speed for east, south, west and north winds.

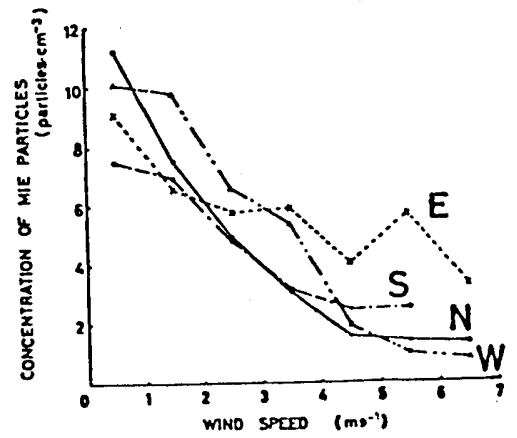

Fig.5. Mie particle concentrations versus wind speed for east, south, west and north winds. 
conclusion can be drawn:

Unless the strong aerosol source exists on the windward, the Mie particle concentration decreases and the small ion concentration increases with the increment of the wind speed. The dependence of the Mie particle concentration on the wind speed is very marked and the high concentration does not occur when the wind speed exceeds $4 \mathrm{~ms}^{-1}$. The Aitken particle concentration is generally insensitive with the variation of the wind speed and tends to increase when a strongly polluted area situates on the windward.

4-2. The extremely high peak values of small ion concentrations recorded on 118 of the whole observation days are associated with strong wind and with very low levels of both Aitken and Mie particle concentrations.

Acknowledgments The authors thank the Saitama Institute of Environmental Pollution for providing plenty of data for wind.

\section{References}

Ikegami, et al., on the meso-scale distribution and the variation characteristics of aerosols (6 and 7th Report), Proc. Soc. Atmos. Elect. Japan, 22, 100-103, $1979 ; 23,48-51,1980$ (in Japanese).

Kobayashi, A., F. Matsumoto and N. Kitagawa, on the relation of aerosols to wind and chemical air pollution during summer season at Urawa-City, ibid. 23, 44-47.

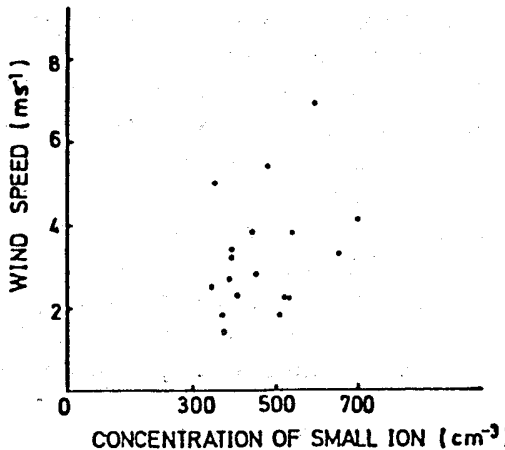
Fig.6. Wind speed versus high small ion concentration peaks.

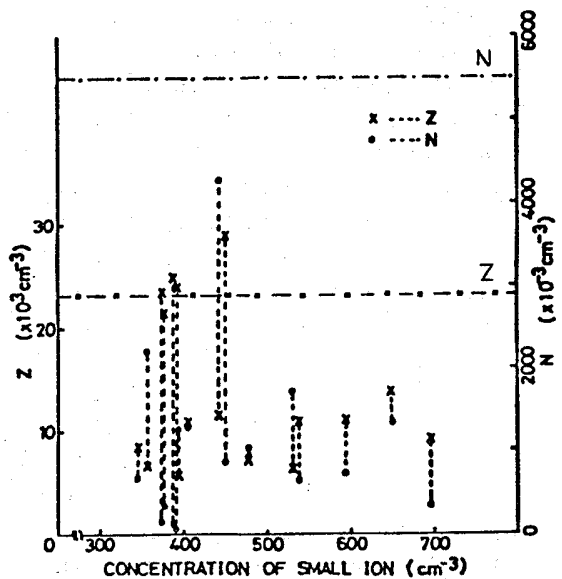

Fig. 7. Plot of Aitken and Mie particle concentrations associated with high small ion concentraion peaks. 1980 (in Japanese).

Misaki, M., On the behaviour of atmospheric aerosols, Kisho-kenkyu Note, 142, in press, 1981 (in Japanese).

(Received March 19, 1981; revised June 28, 1981; accepted June 30,1981 ) 